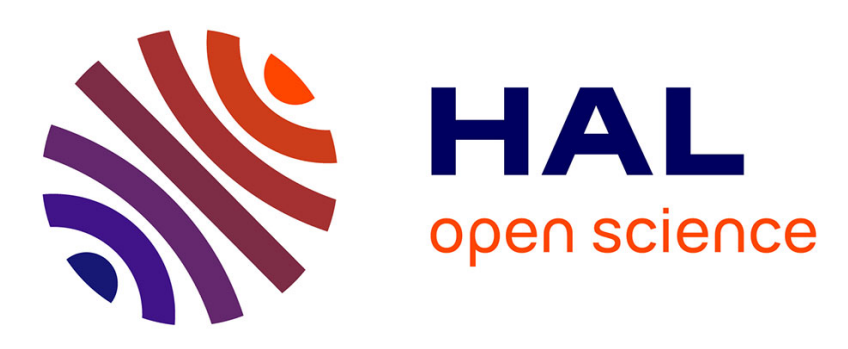

\title{
Partial ordering and phase elasticity in the MnGe short-period helimagnet
}

N. Martin, I. Mirebeau, C. Franz, G. Chaboussant, L. Fomicheva, N. Fomicheva, A. Tsvyashchenko

\section{- To cite this version:}

N. Martin, I. Mirebeau, C. Franz, G. Chaboussant, L. Fomicheva, et al.. Partial ordering and phase elasticity in the MnGe short-period helimagnet. Physical Review B: Condensed Matter and Materials Physics (1998-2015), 2019, 99, pp.100402. 10.1103/PhysRevB.99.100402 . cea-02074769

\section{HAL Id: cea-02074769 https://hal-cea.archives-ouvertes.fr/cea-02074769}

Submitted on 20 Mar 2019

HAL is a multi-disciplinary open access archive for the deposit and dissemination of scientific research documents, whether they are published or not. The documents may come from teaching and research institutions in France or abroad, or from public or private research centers.
L'archive ouverte pluridisciplinaire HAL, est destinée au dépôt et à la diffusion de documents scientifiques de niveau recherche, publiés ou non, émanant des établissements d'enseignement et de recherche français ou étrangers, des laboratoires publics ou privés. 


\title{
Partial ordering and phase elasticity in the MnGe short-period helimagnet
}

\author{
N. Martin, ${ }^{1,}{ }^{*}$ I. Mirebeau, ${ }^{1}$ C. Franz,,${ }^{2,3}$ G. Chaboussant, ${ }^{1}$ L. N. Fomicheva, ${ }^{4}$ and A. V. Tsvyashchenko ${ }^{4}$ \\ ${ }^{1}$ Laboratoire Léon Brillouin, CEA, CNRS, Université Paris-Saclay, CEA Saclay, 91191 Gif-sur-Yvette, France \\ ${ }^{2}$ Heinz Maier-Leibnitz Zentrum, Technische Universität München, 85748 Garching, Germany \\ ${ }^{3}$ Physik-Department E21, Technische Universität München, 85748 Garching, Germany \\ ${ }^{4}$ Vereshchagin Institute for High Pressure Physics, Russian Academy of Sciences, 142190 Troitsk, Moscow, Russia
}

(Received 24 January 2019; published 13 March 2019)

\begin{abstract}
We study the helimagnetic ground state of the MnGe cubic alloy using small-angle neutron scattering and a high-resolution method, the so-called MIEZE spectroscopy. Upon cooling below the Néel temperature $T_{\mathrm{N}}=170(5) \mathrm{K}$, we observe the proliferation of long-wavelength gapless spin fluctuations, concomitant with a continuous evolution of the helical correlation length. These fluctuations disappear at $T_{\text {com }}=32(5) \mathrm{K}$ when the helical period becomes commensurate with the lattice. We propose to describe this intermediate phase as a soliton lattice, promoting nonlinear collective modes, or phasons, over a large temperature interval. We discuss the possible relevance of our results to the previously observed magnetotransport anomalies.
\end{abstract}

DOI: 10.1103/PhysRevB.99.100402

Introduction. Helical magnets with noncentrosymmetric crystal structures-such as $\mathrm{MnSi}$ and $\mathrm{FeGe}$ - are currently a major research topic in condensed-matter physics. On one hand, they host field-induced skyrmion lattices (SkLs) in well-defined pockets of their temperature and magnetic-field $(T, H)$ phase diagrams either in bulk samples [1] or in thin films $[2,3]$. On the other hand, they show quantum critical points at low temperatures under hydrostatic pressure, associated with non-Fermi-liquid behavior and partial ordering (PO) of fluctuating magnetic moments [4,5]. Interestingly, the SkL and PO phases both show a topological Hall effect (THE) [6] usually observed in narrow intervals of a few kelvins nearby the ordering temperature [7-9]. This suggests that the emergence of nontrivial spin textures preceding the suppression of the long-range magnetic order by temperature or pressure is a general phenomenon [6]. These textures are expected to support low-energy excitations, which likely play a crucial role in the anomalous transport properties. However, their direct observation has remained elusive even in perfect single crystals. This might be due to the small moments (typically $\leqslant 1 \mu_{\mathrm{B}}$ per ion) and rather long helimagnetic periods (in the 10-100-nm range) displayed by the studied compounds.

In turn, the MnGe alloy—an isostructural analog of $\mathrm{MnSi}$ and $\mathrm{FeGe}$-appears as a promising candidate for the observation of nontrivial low-energy excitations. It displays a large ordered moment ( $m_{0} \approx 1.9 \mu_{\mathrm{B}}$ per Mn ion at $\left.T=1.5 \mathrm{~K}[10]\right)$ and a very short period $\left(\lambda_{\mathrm{h}} \approx 2.9 \mathrm{~nm}\right.$ at $\left.T=1.5 \mathrm{~K}[11]\right)$, which varies by a factor of $\approx 5 / 3$ as a function of temperature. $\lambda_{\mathrm{h}}$ adopts an incommensurate value for $T_{\text {com }}=32(5) \mathrm{K} \leqslant$ $T \leqslant T_{\mathrm{N}}$ then locks into a commensurate state $\left(\lambda_{\mathrm{h}}=6 a\right.$, where $a$ is the cubic lattice constant of $\mathrm{MnGe}$ ) for $T \lesssim T_{\text {com }}$ [Figs. 1(a) and 1(b)]. This feature contrasts with $\mathrm{MnSi}$ and FeGe for which $\lambda_{h}$ is fairly temperature independent and

\footnotetext{
*nicolas.martin@cea.fr
}

remains incommensurate down to the lowest temperatures. Actually, the small value of $\lambda_{\mathrm{h}}$ in MnGe cannot be explained by a bare competition between a ferromagnetic (FM) exchange and the Dzyaloshinskii-Moriya interactions [12] as for $\mathrm{MnSi}$ or $\mathrm{FeGe}$ since it would require an unphysically large spin-orbit coupling [13]. MnGe thus shows a more complex behavior, close to that of frustrated helimagnets where FM and antiferromagnetic interactions compete [14-16]. It shows magnetic anomalies over an extended pressure range $[10,17]$ as well as a sign change in the anomalous Hall effect (AHE) at $\approx 30 \mathrm{~K}$, yielding a large THE $[18,19]$. Although the Néel temperature of $\mathrm{MnGe}$ is high $\left[T_{\mathrm{N}}=170(5) \mathrm{K}\right]$, anomalous spin fluctuations persist down to low temperatures [20,21]. Up to now, the nature of this peculiar spin dynamics has not been fully clarified since it has mostly been studied using local probes, namely, Mössbauer spectroscopy [20] and muon spin rotation ( $\mu \mathrm{SR})$ [21].

Here, we use a high-resolution neutron-scattering technique, the so-called MIEZE spectroscopy [22], to probe the gapless spin excitations of MnGe at the helical Bragg peak

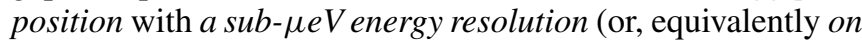
the nanosecond timescale). We observe them in the large temperature interval $T_{\text {com }} \leqslant T \leqslant T_{\mathrm{N}}$. These excitations, which cannot be confused with usual spin waves, suggest that the incommensurate helical order has a finite lifetime. We interpret them as nonlinear collective modes associated with the phase degeneracy of the incommensurate magnetic structure, namely, phasons. These phasons evolve towards diffusive incoherent modes in the vicinity of $T_{\mathrm{N}}$, whereas they disappear below $T_{\text {com }}$ when a commensurate order sets in. We discuss their possible implication in the anomalous magnetotransport properties of MnGe.

Experimental results. We have studied a $\mathrm{MnGe}$ powder sample from the same sample batch as in Refs. [10,17,20,21], synthesized under high pressure and high temperature [23] by combining small-angle neutron scattering (SANS) with MIEZE spectroscopy (see the Supplemental Material [24]). 

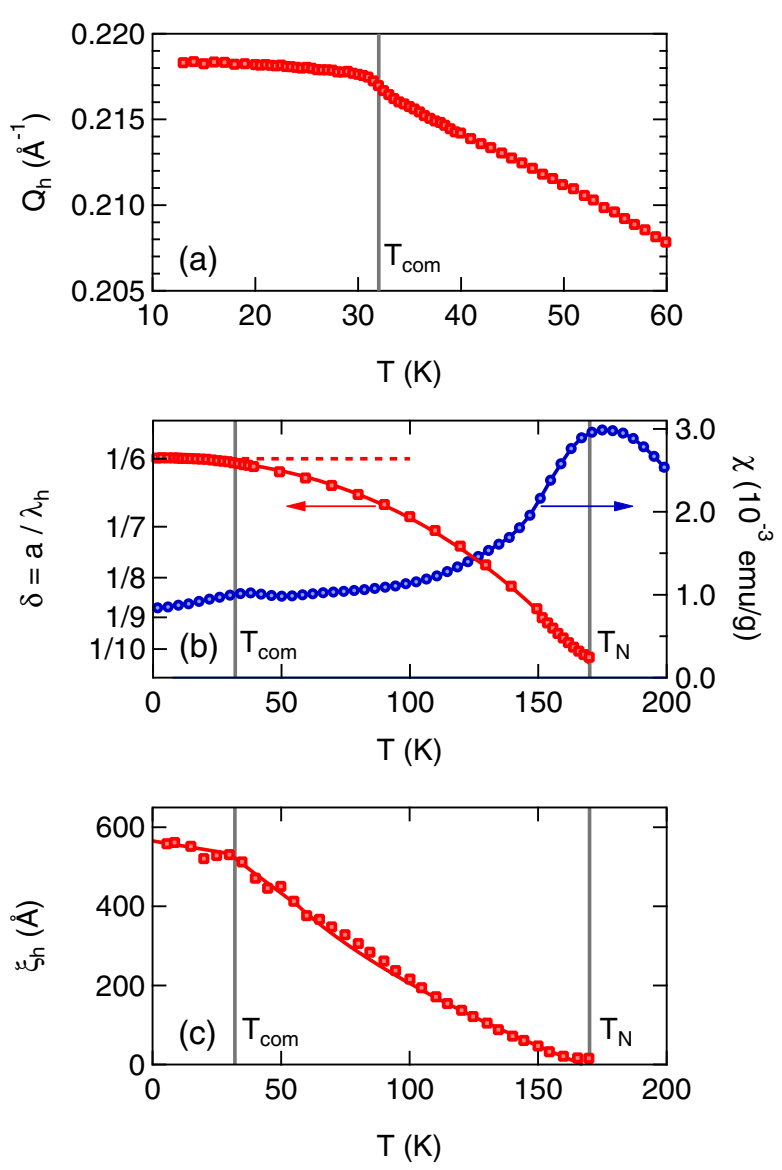

FIG. 1. (a) Temperature dependence of the spin helix propagation wave-vector $Q_{\mathrm{h}}$. (b) Temperature dependence of $\delta=a / \lambda_{\mathrm{h}}$ (where $a$ is the cubic lattice constant of MnGe) and of the zero-fieldcooled homogeneous magnetic susceptibility $\chi$ in a field of 100 Oe. (c) Temperature dependence of the helical correlation length $\xi_{\mathrm{h}}$.

SANS was used to monitor the temperature dependence of the magnetic structure factor $S(Q)$. The experiment has been performed on the PA20 instrument (Laboratoire Léon Brillouin, France [25]). Helimagnetic correlations are observed as a peak in the structure factor $S(Q)$ at a finite momentum transfer $Q_{\mathrm{h}}$, in agreement with previous neutron-diffraction studies $[10,11,18,26]$. Data analysis is carried out using a normalized Lorentzian scattering function,

$$
\mathcal{S}(Q)=\frac{a_{\mathrm{h}}\left(\pi \xi_{\mathrm{h}}\right)^{-1}}{\xi_{\mathrm{h}}^{-2}+\left(Q-Q_{\mathrm{h}}\right)^{2}},
$$

numerically convolved with the resolution function $\mathcal{R}(Q)$ of the spectrometer (see the Supplemental Material [24]). Equation (1) contains the essential information concerning the helimagnetic order, namely, the integrated peak intensity $a_{\mathrm{h}}$ and position $Q_{\mathrm{h}}=2 \pi / \lambda_{\mathrm{h}}$ as well as the helical correlation length $\xi_{\mathrm{h}}$. The peak intensity $a_{\mathrm{h}}$ increases, such as the squared ordered magnetic Mn moment upon cooling the sample below $T_{\mathrm{N}}$, whereas $Q_{\mathrm{h}}$ saturates to a value of $\approx 0.218 \AA^{-1}$ below $T_{\text {com }}=32(5) \mathrm{K}\left[\right.$ Fig. 1(a)] such that the quantity $\delta=a / \lambda_{\mathrm{h}}$ becomes close to $1 / 6$ [Fig. 1(b)]. The correlation length $\xi_{\mathrm{h}}$ displayed in Fig. 1(c) shows a continuous increase upon cooling below $T_{\mathrm{N}}$, at odds with expectations for a three-dimensional
(3D) ordered system for which a sharp increase is expected upon entering the ordered phase. Below $T_{\text {com }}$, $\xi_{\text {h }}$ saturates at $\approx 550 \AA$, close to the resolution limit. The fact that $\mathrm{MnGe}$ does not fully order below $T_{\mathrm{N}}$ strongly suggests the existence of peculiar spin fluctuations [26].

In order to study them directly, we have used MIEZE spectroscopy [22], a high-resolution method which operates optimally in the small-angle regime [27] and maintains high-energy resolution irrespective of the sample under study [28] or its environment [29] since all spin manipulations are performed upstream. Of special interest here, MIEZE can work on a powder helimagnet, such as our MnGe sample, whereas standard neutron spin echo would strongly suffer from the depolarization of the incoming neutron beam by the randomly oriented helimagnetic domains. MIEZE renders the normalized intermediate-scattering function $\mathcal{S}_{\text {norm }}(\mathbf{Q}, \tau)=$ $\mathcal{S}(\mathbf{Q}, \tau) / \mathcal{S}(\mathbf{Q}, 0)$, which is the space-Fourier transform of the van Hove correlation function $\mathcal{G}(\mathbf{r}, \tau)$. Accounting for the selection rule of magnetic scattering, $\mathcal{S}_{\text {norm }}(\mathbf{Q}, \tau)$ is related to the magnetic moment component perpendicular to the scattering vector $\mathbf{m}_{\perp} \equiv \mathbf{m}-(\mathbf{m} \cdot \mathbf{Q}) \cdot \mathbf{Q}=\mathbf{Q} \times(\mathbf{m} \times \mathbf{Q})$. We have measured $\mathcal{S}_{\text {norm }}(\mathbf{Q}, \tau)$ at the position of the helical Bragg peak $|\mathbf{Q}|=Q_{\mathrm{h}}$ in the $T=5-160-\mathrm{K}$ range on the RESEDA spectrometer (Heinz Maier-Leibnitz Institute, Germany [30]). By focusing on this $Q$ value, we are able to detect the fluctuations of $\mathbf{m}_{\perp}$, involving spin components within the helical plane, which are relevant on the length scale of the helical modulation. Assuming that the measured signal corresponds to a superposition of a static (Bragg) contribution and a quasielastic (i.e., centered around zero energy transfer) Lorentzian fluctuation spectrum leads to

$$
\mathcal{S}_{\text {norm }}\left(Q_{\mathrm{h}}, \tau\right)=f \exp \left(-\Gamma_{\mathrm{h}} \tau\right)+(1-f),
$$

where $f$ (respectively, $1-f$ ) is the fluctuating (respectively, static) fraction of the scattering function and $\Gamma_{\mathrm{h}}$ is the inverse characteristic lifetime of the fluctuations. A fit of Eq. (2) to the data of Fig. 2(a) yields the parameters displayed in Figs. 2(b) and 2(c). One immediately observes that $f$ and $\Gamma_{\mathrm{h}}$ acquire nonzero values above $T_{\text {com }}$, whereas they both collapse below $T_{\text {com }}$. Thus, although the helical order appears static on the nanosecond timescale for $T \leqslant T_{\text {com }}$, in agreement with the low-temperature Mössbauer spectroscopy [20] and $\mu$ SR [21] data, gapless spin fluctuations build up in the whole $T_{\text {com }} \leqslant$ $T \leqslant T_{\mathrm{N}}$ range. Their observation at the Bragg peak position together with their disappearance at $T_{\text {com }}$ clearly point towards a dynamics being specific to the incommensurate order.

Taken together, the SANS and MIEZE results show that MnGe only partly orders in the $T_{\text {com }} \lesssim T \lesssim T_{\mathrm{N}}$ range. Below $T_{\text {com }}$, the system is fully ordered: The helical wavelength locks into a commensurate value, and the gapless excitations vanish. This discards usual spin waves as a source for the observed line broadening since their damping would yield a constant $\Gamma_{\mathrm{h}}$ down to the lowest temperatures. On the other hand, assuming a local conservation law on a Mn site $\left(\mathrm{m}_{\text {fluct }}^{2}+m_{\text {ord }}^{2}=\right.$ constant), one can compare the temperature dependence of the static fraction with that of the squared ordered magnetic moment measured by diffraction on the same sample. These two quantities agree as shown in Fig. 3(a). We then deduce the effective fluctuating moment, defined by $m_{\text {fluct }}^{2} \sim$ $m_{\text {ord }}^{2} f /(1-f)$ and scaled to the Mn moment measured at low 

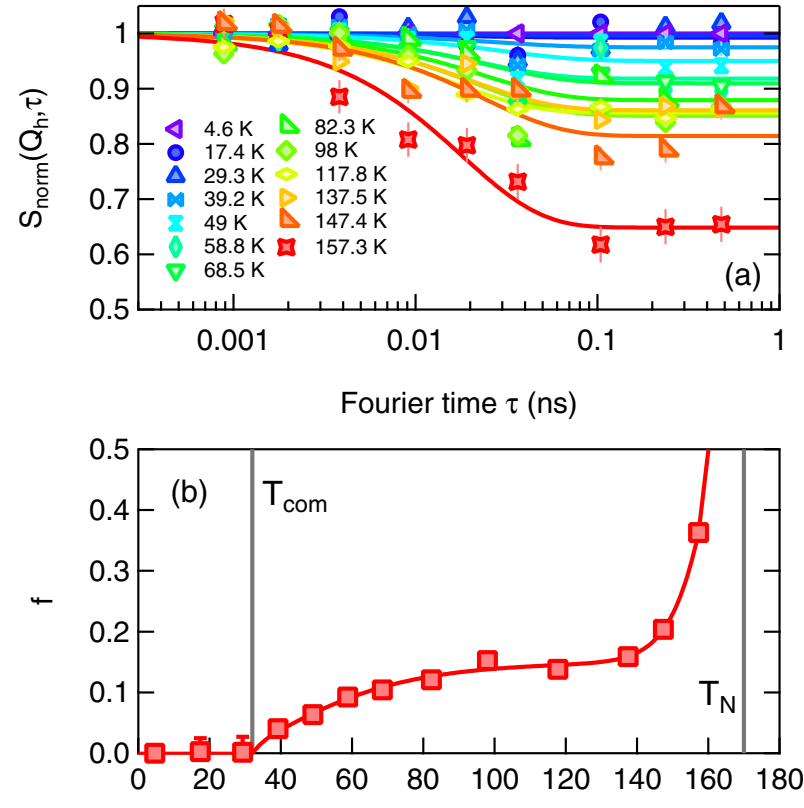

$\mathrm{T}(\mathrm{K})$

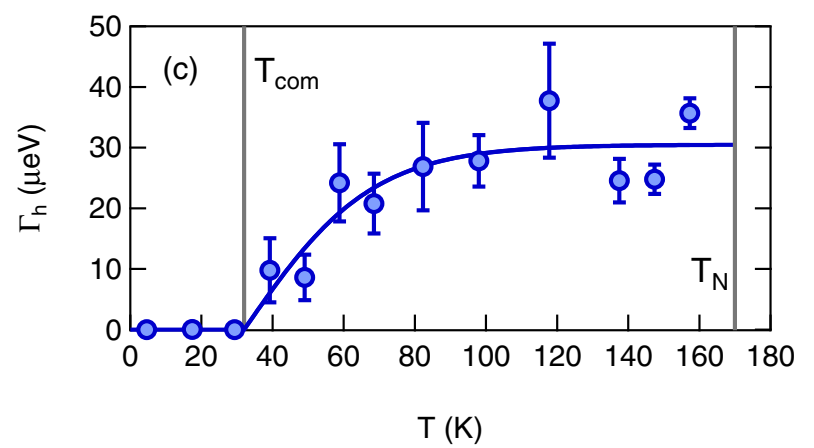

FIG. 2. (a) Normalized intermediate scattering function $\mathcal{S}_{\text {norm }}\left(Q_{\mathrm{h}}, \omega\right)$ measured using MIEZE spectroscopy at the helimagnetic wave-vector $Q_{\mathrm{h}}$ around zero energy transfer. (b) and (c) Parameters extracted from a fit of Eq. (2) to the data, namely, (b) the fluctuating fraction $f$ and (c) linewidth $\Gamma_{\mathrm{h}}$.

temperatures by neutron diffraction. It is almost constant in the incommensurate phase and close to a significant value of $0.5 \mu_{\mathrm{B}} / \mathrm{Mn}$. The angular variation $\alpha$ associated with the fluctuating moment $\left[\tan ^{2} \alpha=f /(1-f)\right]$ is also large on the order of $20^{\circ}$ and becomes comparable to the tilt angle between neighboring spins along the helix pitch $\varphi=2 \pi \delta$ in the vicinity of $T_{\mathrm{N}}$ [Fig. 3(b)]. It will be possible to refine this rough evaluation when an accurate model is proposed for the observed excitations.

Discussion. In incommensurate systems, low-energy excitations are generally called phasons [31], but one should distinguish between linear modes, i.e., soft spin waves occurring in systems with zero in-plane anisotropy, and nonlinear ones. The latter, predicted close to an incommensurate-tocommensurate transition, are usually described as collective excitations associated with the onset of a soliton lattice [32] and may be observed in spatially modulated systems of various kinds. Historically, this picture was developed independently by Dzyaloshinskii [33] to describe the transition
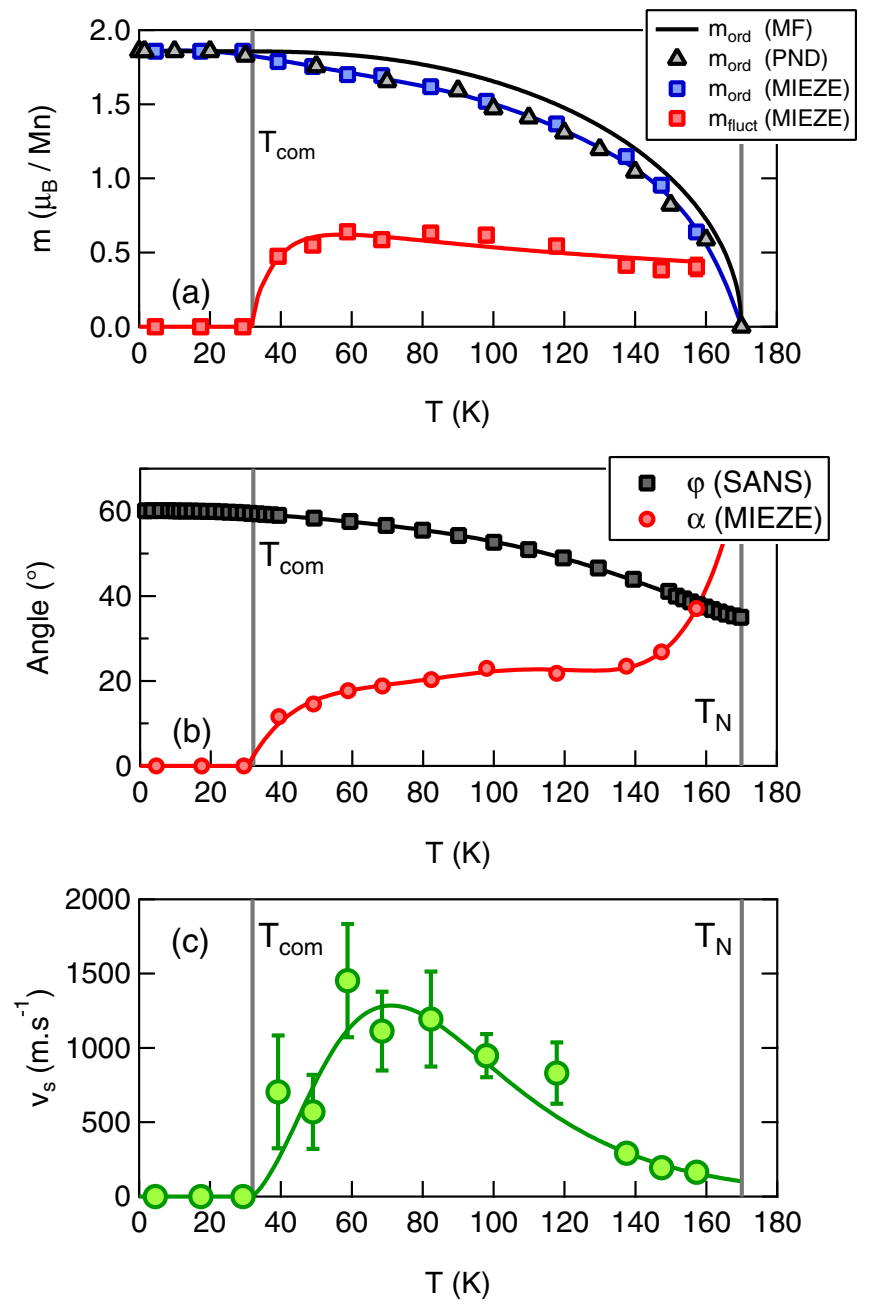

FIG. 3. (a) Ordered $\left(m_{\text {ord }}\right)$ and fluctuating $\left(m_{\text {fluct }}\right)$ moments obtained using MIEZE spectroscopy (squares), compared with powder neutron diffraction [(PND), triangles] refined values (from Ref. [10]) and mean-field [(MF), line] calculation (see the Supplemental Material [24] and Ref. [40]). (b) Angular variation of the local magnetic moment $\alpha$ compared with the tilt angle between neighboring spins along the helix pitch $\varphi=2 \pi \delta$, where $\delta$ is taken from Fig. 1(b). (c) Temperature dependence of the soliton velocity $v_{\mathrm{s}}=\xi_{\mathrm{h}} \Gamma_{\mathrm{h}} / \hbar$ in the free soliton model.

from helical to ferromagnetic order and by de Gennes [34] to model the alignment of cholesteric liquid crystals by a magnetic field. It also explains supersonic vibrations in a compound with flexible crystal structure [35]. Solitons are mobile domain walls between essentially commensurate domains, and their lattice describes the continually accumulating phase shifts near the transition to commensurate order. The associated excitation, or phason, is bound to the breaking of translational invariance and therefore manifests itself as a gapless mode only in the incommensurate phase.

In helical magnets, anisotropy distorts the helical arrangement, and the angular variation of the magnetic moment is described by the sine-Gordon equation for a soliton lattice [36], which justifies the above picture. Direct observations of the magnetic soliton lattice in $3 \mathrm{D}$ compounds are rare but can be found in erbium [37] or more recently in $\mathrm{CuB}_{2} \mathrm{O}_{4}$ [38]. 
In MnGe, although there is no direct evidence for a soliton lattice from the diffraction data (probably hampered by the powder average), we propose to describe the observed excitations accordingly: Nonlinear phasons propagate, such as Goldstone modes [39]-i.e., at zero energy cost—and limit the helimagnetic order lifetime to $\tau_{\mathrm{h}} \approx 20 \mathrm{ps}$ (namely, $\tau_{\mathrm{h}}=0.659 / \Gamma_{\mathrm{h}}$ where $\Gamma_{\mathrm{h}}$ is expressed in $\mu \mathrm{eV}$ and $\tau_{\mathrm{h}}$ in nanoseconds) whereas preserving its overall periodicity. They disappear below $T_{\text {com }}$, most likely at the expense of gapped excitations when the commensurate helical order is pinned along anisotropy directions. The soliton lattice can be preserved well above $T_{\text {com }}$ owing to the strong anisotropy of $\mathrm{MnGe}$, deduced from the large value of the upper critical field $H_{C 2}$, above which the system reaches a fully polarized state $(\approx 10 \mathrm{~T}$ at $30 \mathrm{~K}[18])$. It must however collapse at high temperatures when the strong increase in the fluctuating fraction of $\mathcal{S}_{\text {norm }}\left(Q_{\mathrm{h}}, \omega\right)$ suggests the destruction of the order, mediated by paramagnetic excitations.

Alternatively, assuming independent solitons, one could estimate their velocity by making a crude analogy with onedimensional magnets where their propagation has been studied in detail [41-43]. In this picture, the inverse correlation length $\xi_{\mathrm{h}}^{-1}$ measures the soliton density, whereas the inverse lifetime $\Gamma_{\mathrm{h}}^{-1}$ measures the flipping rate of the helimagnetic state due to the passage of solitons through the ordered domains. In the absence of intrinsic damping, one can obtain a lower estimate for their average velocity via $v_{\mathrm{s}}=\xi_{\mathrm{h}} \Gamma_{\mathrm{h}} / \hbar$, using data of Figs. 1(c) and 2(c). $v_{\mathrm{s}}$ displays a maximum value at $\approx 1300 \mathrm{~m} \mathrm{~s}^{-1}$ at $T \approx 70 \mathrm{~K}$ and drops down to zero at both edges of the incommensurate phase [Fig. 3(c)].

We now shortly discuss the possible implications of such excitations on the magnetotransport properties of MnGe. Strikingly, the commensurate-incommensurate transition at $T_{\text {com }}$ occurs concomitantly with a change in sign of the AHE versus temperature $[18,19]$. It is therefore likely that the low-energy excitations observed here participate in the resulting THE. In Ref. [19], it was recently proposed that spin fluctuations of locally correlated moments give rise to another anomalous Hall effect with the opposite sign to the THE. The suppression of such excitations at the commensurate transition could then explain the observed change in sign. A lattice of topological defects, persisting in the zero magnetic field, has also been proposed to interpret the elastic and magnetotransport anomalies [44]. Our experiments do not allow distinguishing between the domain-wall picture (proposed here) and the monopole picture discussed in Ref. [44]. But the observation of nonlinear modes could explain a THE, whereas the linear phason modes present in unpinned helixes could not. Indeed, even without well-defined lattices, solitons might be perceived as a local Berry curvature by the conduction electrons, which are moving on timescales much smaller than the nanosecond one. Such a mechanism could be at play in the region of $T_{\mathrm{N}}$ in MnGe. Here too, the contribution of the lowenergy mode to the THE is expected to be sizable, owing to the large value of the effective fluctuating moment. It would be actually interesting to follow the evolution of the low-energy mode with the applied field to check the above framework. It should disappear upon reaching the upper critical field $H_{C 2}$, a region which can be conveniently explored using MIEZE spectroscopy.

Conclusions. To summarize, we have observed a gapless magnetic mode in the MnGe helical magnet, solely stabilized in the incommensurate phase. We interpret it as a nonlinear phason mode linked with the onset of a soliton lattice. This mode disappears at the transition towards commensurate order and may transform as individual solitonslike excitations in the region of $T_{\mathrm{N}}$. It was observed in a powder sample using a high-resolution spectroscopic method. It is expected to be quite general in spin helix systems due to the pinning by anisotropy, but its observation was possible in MnGe thanks to the strong anisotropy, high moment value, and small helical pitch, which ensures a high density of excitations. It likely plays a crucial role in the giant topological Hall effect shown by this compound.

Acknowledgments. We are grateful to A. Goukasov and U. $\mathrm{K}$. Rößler for enlightening discussions at the early stage of this Rapid Communication and to Y. Sidis and S. Petit for their critical reading of the Rapid Communication and many useful suggestions. We also thank A. Forget for sample conditioning, A. Mantwill for preparing the neutron sample holder for the MIEZE experiment, and M. Detrez and S. Gautrot for setting up the SANS experiment. This Rapid Communication is based upon experiments performed at the PA20 instrument operated by Laboratoire Léon Brillouin, Gif-sur-Yvette, France and at the RESEDA instrument operated by FRM II at the Heinz Maier-Leibnitz Zentrum, Garching, Germany. A.V.T. is grateful to the Russian Science Foundation (Grant No. 17-12-01050).
[1] S. Mühlbauer, B. Binz, F. Jonietz, C. Pfleiderer, A. Rosch, A. Neubauer, R. Georgii, and P. Böni, Science 323, 915 (2009).

[2] X. Z. Yu, N. Kanazawa, Y. Onose, K. Kimoto, W. Z. Zhang, S. Ishiwata, Y. Matsui, and Y. Tokura, Nature Mater. 10, 106 (2011)

[3] S. Seki, X. Z. Yu, S. Ishiwata, and Y. Tokura, Science 336, 198 (2012).

[4] C. Pfleiderer, D. Reznik, L. Pintschovius, H. v. Lohneysen, M. Garst, and A. Rosch, Nature (London) 427, 227 (2004).

[5] A. Barla, H. Wilhelm, M. K. Forthaus, C. Strohm, R. Rüffer, M. Schmidt, K. Koepernik, U. K. Rößler, and M. M. Abd-Elmeguid, Phys. Rev. Lett. 114, 016803 (2015).
[6] R. Ritz, M. Halder, M. Wagner, C. Franz, A. Bauer, and C. Pfleiderer, Nature (London) 497, 231 (2013).

[7] H. Wilhelm, M. Baenitz, M. Schmidt, U. K. Rößler, A. A. Leonov, and A. N. Bogdanov, Phys. Rev. Lett. 107, 127203 (2011).

[8] M. Janoschek, M. Garst, A. Bauer, P. Krautscheid, R. Georgii, P. Böni, and C. Pfleiderer, Phys. Rev. B 87, 134407 (2013).

[9] L. J. Bannenberg, K. Kakurai, P. Falus, E. Lelièvre-Berna, R. Dalgliesh, C. D. Dewhurst, F. Qian, Y. Onose, Y. Endoh, Y. Tokura et al., Phys. Rev. B 95, 144433 (2017).

[10] M. Deutsch, O. L. Makarova, T. C. Hansen, M. T. FernandezDiaz, V. A. Sidorov, A. V. Tsvyashchenko, L. N. Fomicheva, 
F. Porcher, S. Petit, K. Koepernik et al., Phys. Rev. B 89, 180407(R) (2014).

[11] O. L. Makarova, A. V. Tsvyashchenko, G. André, F. Porcher, L. N. Fomicheva, N. Rey, and I. Mirebeau, Phys. Rev. B 85, 205205 (2012).

[12] J. Kishine and A. Ovchinnikov, in Solid State Physics, edited by R. E. Camley and R. L. Stamps (Academic Press, San Diego, 2015), Vol. 66, pp. 1-130.

[13] V. A. Chizhikov and V. E. Dmitrienko, Phys. Rev. B 88, 214402 (2013).

[14] J. Villain, J. Phys. Chem. Solids 11, 303 (1959).

[15] A. Yoshimori, J. Phys. Soc. Jpn. 14, 807 (1959).

[16] Y. A. Izyumov, Sov. Phys. Usp. 27, 845 (1984).

[17] N. Martin, M. Deutsch, J.-P. Itié, J.-P. Rueff, U. K. Rössler, K. Koepernik, L. N. Fomicheva, A. V. Tsvyashchenko, and I. Mirebeau, Phys. Rev. B 93, 214404 (2016).

[18] N. Kanazawa, Y. Onose, T. Arima, D. Okuyama, K. Ohoyama, S. Wakimoto, K. Kakurai, S. Ishiwata, and Y. Tokura, Phys. Rev. Lett. 106, 156603 (2011).

[19] H. Ishizuka and N. Nagaosa, Sci. Adv. 4, eaap9962 (2018).

[20] M. Deutsch, P. Bonville, A. V. Tsvyashchenko, L. N. Fomicheva, F. Porcher, F. Damay, S. Petit, and I. Mirebeau, Phys. Rev. B 90, 144401 (2014).

[21] N. Martin, M. Deutsch, F. Bert, D. Andreica, A. Amato, P. Bonfà, R. De Renzi, U. K. Rößler, P. Bonville, L. N. Fomicheva et al., Phys. Rev. B 93, 174405 (2016).

[22] R. Gähler, R. Golub, and T. Keller, Physica B 180-181, 899 (1992).

[23] A. Tsvyashchenko, V. Sidorov, L. Fomicheva, V. Krasnorussky, R. Sadykov, J. Thompson, K. Gofryk, F. Ronning, and V. Ivanov, in Magnetism and Magnetic Materials V, Solid State Phenomena (Scientific.Net, Zurich, Switzerland, 2012), Vol. 190, pp. 225-228.

[24] See Supplemental Material at http://link.aps.org/supplemental/ 10.1103/PhysRevB.99.100402 for details about sample preparation and characterization, experimental techniques, and mean-field calculations, which includes Refs. [45-47].

[25] G. Chaboussant, S. Désert, P. Lavie, and A. Brûlet, J. Phys.: Conf. Ser. 340, 012002 (2012).

[26] E. Altynbaev, S.-A. Siegfried, V. Dyadkin, E. Moskvin, D. Menzel, A. Heinemann, C. Dewhurst, L. Fomicheva,
A. Tsvyashchenko, and S. Grigoriev, Phys. Rev. B 90, 174420 (2014).

[27] N. Martin, Nucl. Instrum. Methods Phys. Res., Sect. A 882, 11 (2018).

[28] J. Kindervater, S. Säubert, and P. Böni, Phys. Rev. B 95, 014429 (2017).

[29] J. Kindervater, N. Martin, W. Häußler, M. Krautloher, C. Fuchs, S. Mühlbauer, J. A. Lim, E. Blackburn, P. Böni, and C. Pfleiderer, EPJ Web Conf. 83, 03008 (2015).

[30] C. Franz and T. Schröder, J. Large-Scale Res. Facilities 1, A14 (2015).

[31] A. W. Overhauser, Phys. Rev. B 3, 3173 (1971).

[32] P. Bak, Rep. Prog. Phys. 45, 587 (1982).

[33] I. Dzyaloshinskii, Sov. Phys. JETP 20, 665 (1965).

[34] P.-G. de Gennes, Solid State Commun. 6, 163 (1968).

[35] M. E. Manley, P. J. Stonaha, D. L. Abernathy, S. Chi, R. Sahul, R. P. Hermann, and J. D. Budai, Nat. Commun. 9, 1823 (2018).

[36] Y. Izyumov, Physica B 174, 9 (1991).

[37] M. Habenschuss, C. Stassis, S. K. Sinha, H. W. Deckman, and F. H. Spedding, Phys. Rev. B 10, 1020 (1974).

[38] B. Roessli, J. Schefer, G. A. Petrakovskii, B. Ouladdiaf, M. Boehm, U. Staub, A. Vorotinov, and L. Bezmaternikh, Phys. Rev. Lett. 86, 1885 (2001).

[39] J. Goldstone, A. Salam, and S. Weinberg, Phys. Rev. 127, 965 (1962).

[40] D. C. Johnston, Phys. Rev. B 91, 064427 (2015).

[41] J. Villain, Physica B+C 79, 1 (1975).

[42] K. Sasaki and T. Tsuzuki, Physica B+C 107, 97 (1981).

[43] J. P. Boucher, L. P. Regnault, J. Rossat-Mignod, Y. Henry, J. Bouillot, and W. G. Stirling, Phys. Rev. B 31, 3015 (1985).

[44] N. Kanazawa, Y. Nii, X. X. Zhang, A. S. Mishchenko, G. De Filippis, F. Kagawa, Y. Iwasa, N. Nagaosa, and Y. Tokura, Nat. Commun. 7, 11622 (2016).

[45] R. Viennois, C. Reibel, D. Ravot, R. Debord, and S. Pailhès, Europhys. Lett. 111, 17008 (2015).

[46] B. Hammouda and D. F. R. Mildner, J. Appl. Crystallogr. 40, 250 (2007).

[47] W. Häussler and U. Schmidt, Phys. Chem. Chem. Phys. 7, 1245 (2005). 


\title{
Supplementary material for
}

"Partial ordering and phase elasticity in the MnGe short period helimagnet"

\author{
N. Martin, ${ }^{1}$ I. Mirebeau,${ }^{1}$ C. Franz,${ }^{2,3}$ G. Chaboussant,${ }^{1}$ \\ L. N. Fomicheva, ${ }^{4}$ and A. V. Tsvyashchenko ${ }^{4}$ \\ ${ }^{1}$ Laboratoire Léon Brillouin, CEA, CNRS, Université Paris-Saclay, \\ CEA Saclay 91191 Gif-sur-Yvette, Franc $\AA^{\circledast}$ \\ ${ }^{2}$ Heinz Maier-Leibnitz Zentrum, Technische Universität München, 85748 Garching, Germany \\ ${ }^{3}$ Physik-Department E21, Technische Universität München, 85748 Garching, Germany \\ ${ }_{4}^{4}$ Vereshchagin Institute for High Pressure Physics, \\ Russian Academy of Sciences, 142190, Troitsk, Moscow, Russia
}

(Dated: January 18, 2019)

In this supplement, we provide details regarding sample preparation and characterization (Sec. I). The experimental methods used to obtain the results exposed in the main text, namely small-angle neutron scattering (Sec. III) and MIEZE spectroscopy (Sec. III) are also described. In Sec. [V] we reproduce the mean-field expressions used to calculate the expectation temperature-dependence of the ordered moment.

\section{SAMPLE PREPARATION AND CHARACTERIZATION}

Polycrystalline MnGe was synthesized under 8 GPa in a toroidal high-pressure apparatus by melting reaction with $\mathrm{Mn}$ and Ge. The purity of the constituents was $99.9 \%$ and $99.999 \%$ for $\mathrm{Mn}$ and Ge respectively. The pellets of well-mixed powdered constituents were placed in rock-salt pipe ampoules and then directly electrically heated to $\mathrm{T} \approx 1600^{\circ} \mathrm{C}$. Then the sample was quenched to room temperature before releasing the applied pressure ${ }^{1}$. It was checked by neutron and $\mathrm{X}$ ray diffraction, showing an amount of impurities smaller than $2 \%$. For the current study, the sample originates from the same batch as for our previous neutron ${ }^{2[3}, \mu \mathrm{SR}^{4}$ and $\mathrm{X}$-ray ${ }^{\sqrt{5}}$ experiments. The powder was conditioned in a cylindrical shape of diameter $3 \mathrm{~mm}$, yielding a mass of about $350 \mathrm{mg}$.

Magnetic susceptibility (Fig. 1b of main text) has been recorded on a small amount $(\sim 7 \mathrm{mg})$ of the same sample using a DynaCool 9T Quantum Design PPMS (Laboratoire Léon Brillouin) and is in excellent agreement with previously reported data ${ }^{1,3617}$. This includes the anomaly seen around $30 \mathrm{~K}$ which is attributed to the commensurate-incommensurate transition in the present work. 


\section{SMALL-ANGLE NEUTRON SCATTERING}

The reported Small-Angle Neutron Scattering (SANS) experiment took place at the novel beamline PA2 ${ }^{8}$ installed in the guide hall of the Orphée reactor (Laboratoire Léon Brillouin). We have used a neutron beam prepared by a mechanical velocity selector, yielding a mean wavelength $\lambda_{0}=4.46 \AA$ and a bandwidth of full width at half maximum $\Delta \lambda / \lambda_{0}=11.7 \%$. Sample-to-detector distance was set to $1.88 \mathrm{~m}$ in order to cover the momentum tranfser $(Q)$-range required to study the whole temperature dependence of the helical order over the $5 \leq T \leq T_{\mathrm{N}} \simeq 170 \mathrm{~K}$ range. Collimation length was $1.75 \mathrm{~m}$, offering an optimal trade-off between incoming flux and momentum transfer resolution. Selected data is displayed in Fig. 1.
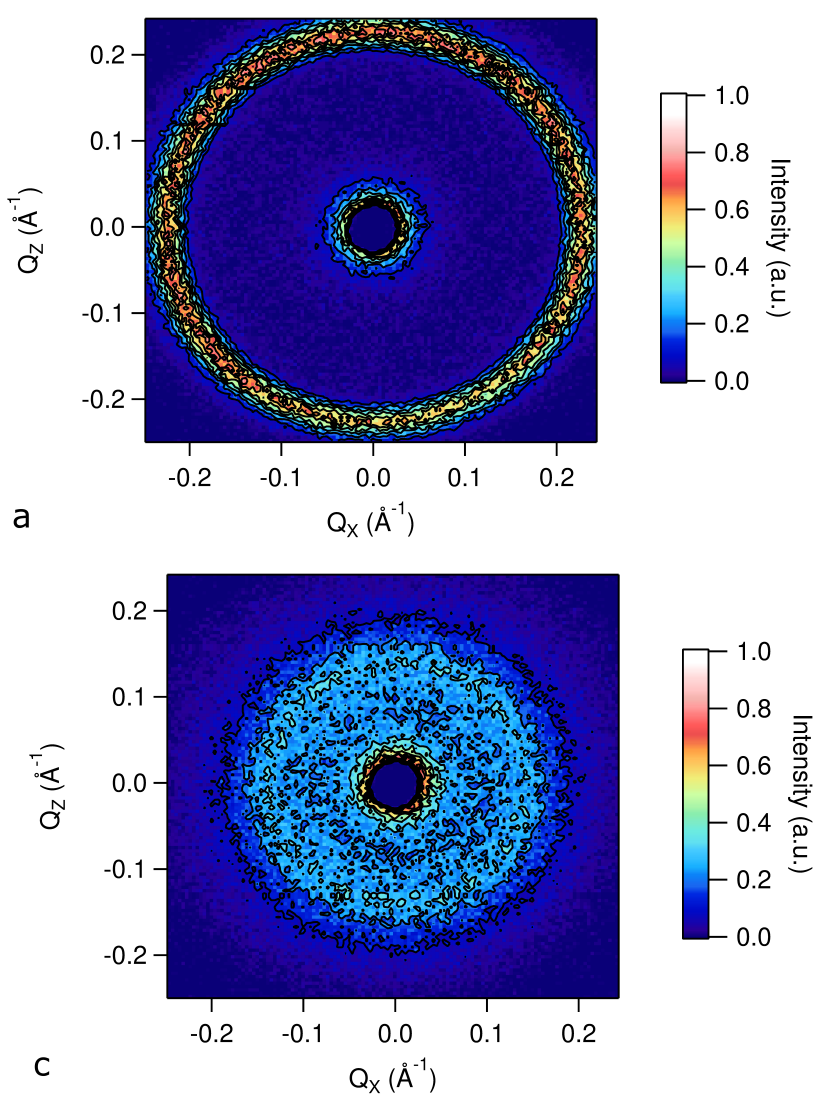
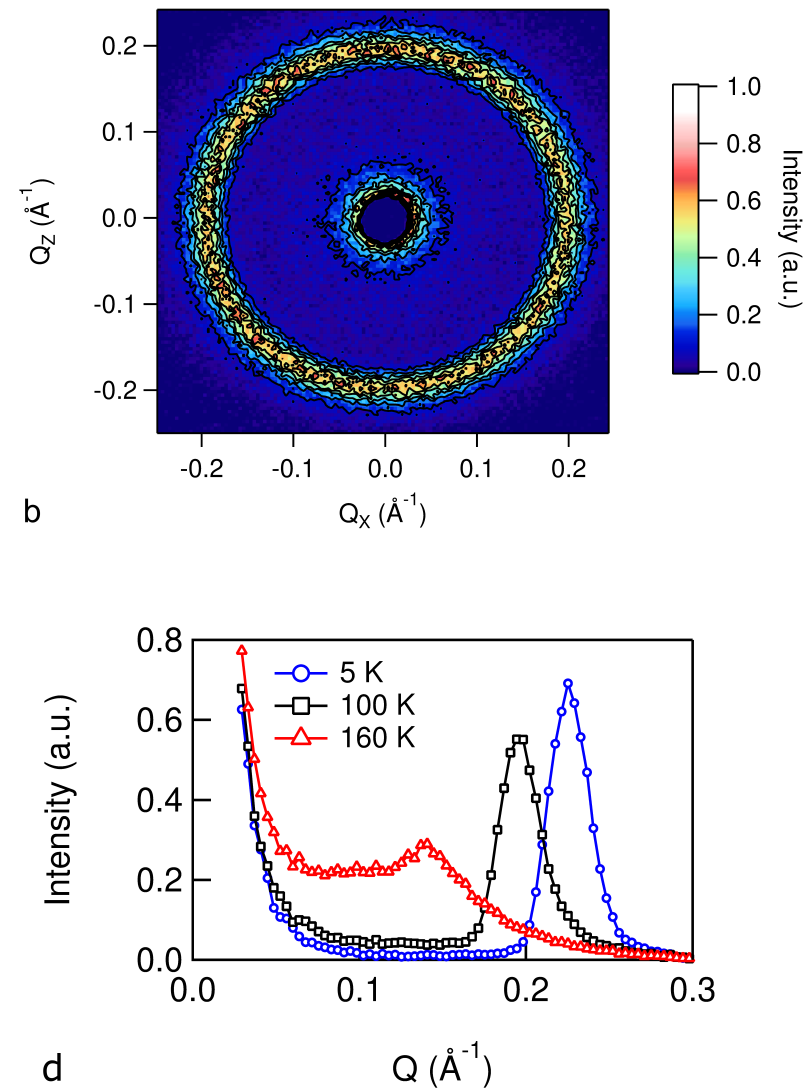

FIG. 1: (a-c) SANS maps recorded on the PA20 instrument at $\mathrm{T}=5 \mathrm{~K}(\mathbf{a}), 100 \mathrm{~K}$ (b) and $160 \mathrm{~K}$ (c). (d) Radial averages of the signals displayed in the previous panels. At the highest temperature, one can note the onset of a step-like contribution at low Q, as reported previously in Ref. 9 .

Data analysis was carried out using the scattering function

$$
\mathcal{S}(Q)=\frac{a_{\mathrm{bg}}}{q^{\mathrm{bg}}}+\frac{a_{\mathrm{h}} \cdot\left(\pi \xi_{\mathrm{h}}\right)^{-1}}{\xi_{\mathrm{h}}^{-2}+\left(Q-Q_{\mathrm{h}}\right)^{2}}
$$


numerically convolved with the Gaussian instrumental resolution function $\mathcal{R}(Q)$ calculated following Ref. 10. In Eq. 1, the first term accounts for small angle signal (nuclear background superimposed with ferromagnetic correlations of increasing intensity upon heating) while the second one corresponds to the helical satellite defining domains of spin spirals with a spatial periodicity $\lambda_{\mathrm{h}}=2 \pi / Q_{\mathrm{h}}$ and a correlation length $\xi_{\mathrm{h}}$.

\section{MIEZE SPECTROSCOPY}

MIEZE (standing for Modulation of Intensity Emerging with Zero Effort) spectroscopy is an extension of the so-called Neutron Resonant Spin Echo (NRSE) technique due to R. Gähler and R. Golub 11 . It is based on the use of radiofrequency spin flippers (RFSF). RFSFs produce a superposition of a static field $B$ and an oscillating field of angular frequency $\omega_{\mathrm{rf}}$. Both quantities are linked by the resonance condition $\omega_{\mathrm{rf}}=\gamma_{\mathrm{n}} \cdot B$, where $\gamma_{\mathrm{n}}=2 \pi \times 2.916 \mathrm{kHz} \cdot \mathrm{G}^{-1}$ is the neutron gyromagnetic ratio. It can be shown that by the following appropriate choice of distances and oscillating field frequencies (as defined in Fig. 2a)

$$
L_{2}=\frac{L_{1}}{\omega_{\mathrm{rf}, 2} / \omega_{\mathrm{rf}, 1}-1}
$$

one can achieve a harmonic time-modulation of the intensity a polarized neutron beam, if polarization of the latter is analyzed prior to detection. This beam will display the following timedependence:

$$
I\left(t_{\mathrm{d}}\right)=I_{\mathrm{av}} \cdot\left(1+\mathcal{C}_{\mathrm{res}} \cdot \cos \left[\omega_{\mathrm{M}} t_{\mathrm{d}}\right]\right)
$$

where $t_{\mathrm{d}}$ is the detection time, $I_{\mathrm{av}}$ the time averaged beam intensity, $\mathcal{C}_{\text {res }} \leq 1$ an experimental factor accounting for instrumental decay of contrast and $\omega_{\mathrm{M}}=2\left(\omega_{\mathrm{rf}, 2}-\omega_{\mathrm{rf}, 1}\right)$ the modulation frequency. Note that the time-modulation of Eq. 3 can be achieved even using beams presenting large wavelength distributions $\Delta \lambda / \lambda_{0} \approx 10-15 \%$, where $\Delta \lambda$ is the width of the incoming spectrum and $\lambda_{0}$ the mean wavelength.

Let us assume that a neutron of initial velocity $v_{\mathrm{i}}$ (or wavelength $\lambda_{\mathrm{i}}$ ) exchanges a certain amount of energy $\hbar \omega$ upon scattering by the sample (S in Fig. 2a), corresponding to a change of velocity $\delta v$. It will thus experience a time delay in its propagation between the sample and the detector (D in Fig. 2a), which reads

$$
\delta t_{\mathrm{d}}=\delta\left(\frac{L_{\mathrm{SD}}}{v_{\mathrm{i}}}\right)=-L_{\mathrm{SD}} \frac{\delta v}{v_{\mathrm{i}}^{2}}
$$



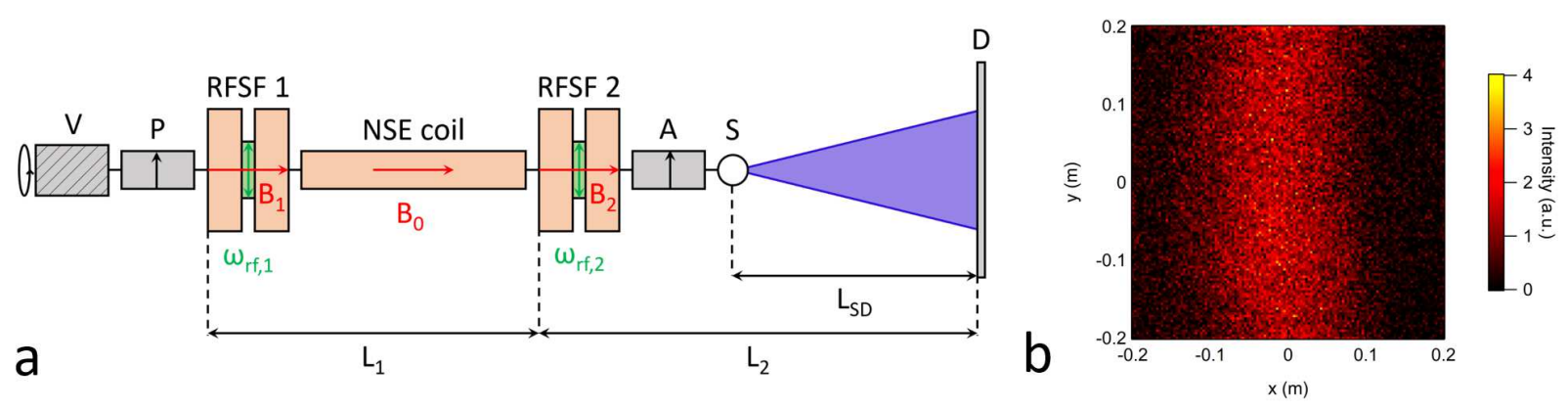

FIG. 2: (a) Schematical MIEZE setup as implemented at RESEDA. Neutrons are coarsely monochromated by a helical velocity selector $(\mathbf{V})$ and are traversing the instrument from left to right. They are polarized using a supermirror-based V-cavity $(\mathbf{P})$. Then, their spin is manipulated by the pair of radiofrequency spin flippers (RFSF1 and RFSF2). This polarization oscillation is converted into an intensity modulation in time by analysis through another (more compact) V-cavity (A). After scattering by the sample (S), neutrons are detected by a high dynamics CASCADE detector which uses thin ${ }^{10} \mathrm{~B}$ foils as conversion layers $(\mathbf{D})$. The bunching signal which is fed to the detector is phase-locked with the generators producing the RF fields, suppressing possible time jitters and smearing out of the data. Note that the setup also include a solenoid (NSE coil) which allows reaching the smallest Fourier times (see text). (b) Portion of the Debye-Scherrer ring due to the helimagnetic Bragg scattering, recorded at $\mathrm{T}=5 \mathrm{~K}$. The ability to detect neutrons over an extended solid angle is a key asset of MIEZE spectroscopy, as opposed to single counter techniques.

where $L_{\mathrm{SD}}$ is the sample-to-detector distance (see Fig. 2a). Using Eq. 4. one can find an expression for the energy transfer

$$
\hbar \omega=\frac{m_{\mathrm{n}}}{2} \cdot\left(v_{\mathrm{i}}^{2}-\left[v_{\mathrm{i}}+\delta v\right]^{2}\right) \underset{\delta v \ll v_{\mathrm{i}}}{\longrightarrow}-m_{\mathrm{n}} v_{\mathrm{i}} \delta v,
$$

where $m_{\mathrm{n}}$ the neutron mass.

Combining Eqs. 4 and 5, one finds an expression for the measured intensity in the case of quasi-elastic scattering $\left(\delta v \ll v_{\mathrm{i}}\right)$

$$
\begin{aligned}
I\left(t_{\mathrm{d}}\right) & =I_{\mathrm{av}} \cdot\left(1+\mathcal{C}_{\mathrm{res}} \cdot \cos \left[\omega_{\mathrm{M}}\left(t_{\mathrm{d}}+\delta t_{\mathrm{d}}\right)\right]\right) \\
& =I_{\mathrm{av}} \cdot\left(1+\mathcal{C}_{\mathrm{res}} \cdot \cos \left[\omega_{\mathrm{M}} t_{\mathrm{d}}-\omega \tau\right]\right),
\end{aligned}
$$

where we have introduced the Fourier time

$$
\tau=\frac{\hbar \omega_{\mathrm{M}} L_{\mathrm{SD}}}{m_{\mathrm{n}} v_{\mathrm{i}}^{3}}=\frac{m_{\mathrm{n}}^{2} \omega_{\mathrm{M}} L_{\mathrm{SD}}}{8 \pi^{3} \hbar^{2}} \cdot \lambda_{\mathrm{i}}^{3}
$$

An actual experimental situation generally implies a distribution of energy transfers. Assuming that this distribution -the dynamical structure factor $\mathcal{S}(Q, \omega)$ - is symmetric with respect to $\omega^{1}$,

\footnotetext{
${ }^{1}$ Such an approximation is valid in classical limit $\Gamma \ll k_{\mathrm{b}} T$, where $\Gamma$ is the characteristic energy width of $\mathcal{S}(Q, \omega)$.
} 
Eq. 6 may be rewritten as follows:

$$
I\left(t_{\mathrm{d}}\right)=I_{\mathrm{av}} \cdot\left(1+\mathcal{C}_{\mathrm{res}} \cdot \cos \left[\omega_{\mathrm{M}} t_{\mathrm{d}}\right] \cdot \frac{\int \mathcal{S}(Q, \omega) \cos [\omega \tau] d \omega}{\int \mathcal{S}(Q, \omega) d \omega}\right),
$$

where the second term within brackets contains the signal contrast

$$
\mathcal{C}=\frac{\int \mathcal{S}(Q, \omega) \cos [\omega \tau] d \omega}{\int \mathcal{S}(Q, \omega) d \omega}=\frac{\mathcal{S}(Q, \tau)}{\mathcal{S}(Q, 0)} \equiv \mathcal{S}_{\text {norm }}(Q, \tau)
$$

which is the measured quantity. $\mathcal{S}_{\text {norm }}(Q, \tau)$ is the real part of the time Fourier transform of the dynamical structure factor (also termed "intermediate scattering function"). The MIEZE signal contrast $\mathcal{C}$ is experimentally obtained by recording $I\left(t_{\mathrm{d}}\right)$ and evaluating $\mathcal{C}=\mathcal{A} / I_{\text {av }}$ where $\mathcal{A}$ is the signal amplitude. The resolution correction $\mathcal{C}_{\text {res }}$ is determined similarly by measuring the signal due to an elastic scatterer i.e. for which a decay in contrast will be solely due to instrumental factors.

The main advantage of MIEZE is -alike NSE- that one can use a coarsely monochromatic beam $\left(\Delta \lambda / \lambda_{0} \geq 10 \%\right)$ while achieving long time resolutions ${ }^{2}$ (Eq. 7 shows that $\tau$ is proportional to $\lambda_{\mathrm{i}}^{3}$ and independent of the incoming beam spectral width). As opposed to conventional neutron spectroscopy techniques, one can thus achieve high resolution without sacrificing most of the beam intensity. Typical resolutions are of the order of several ns, while normally limited to a few ps. Moreover, since beam polarization is analyzed prior to scattering, one can measured dynamic properties of depolarizing systems (such as multidomain ferromagnets ${ }^{12}$ ) or under large magnetic fields $\frac{13}{13}$ without loss of sensitivity. Such properties are also relevant to our MnGe powder samples in which a number of spin helices will abruptly terminate at grain boundaries, leading to disoriented fields within the sample and hence depolarization destroying the $N(R) S E$ signal. Last but not least, it is possible to access very small Fourier times without loss of signal contrast by adding a longitudinal solenoid to the setup ( $\vec{B}_{0}$ in Fig. $\left.2 \mathrm{a}\right)$. This is achieved by making use of the so-called Field-Integral Subtraction Method 14 .

The experiment described in the main text was performed at the high resolution beamline RESEDA $^{\sqrt{15}}$ located in the guide hall of the Heinz Maier-Leibnitz Institute (Garching, Germany). We have used $\lambda_{0}=6.5 \AA$ and $\Delta \lambda / \lambda_{0}=11 \%$. With such settings, Fourier times were accessed in the 1 ps $\lesssim \tau \lesssim 1$ ns range. Resolution correction was performed by measuring $\mathcal{C}_{\text {res }}$ at the helical peak of MnGe at the lowest temperature $(5 \mathrm{~K})$, where all dynamics are assumed to be frozen out on the ns time-scale. In order to account for the well-understood Q-dependence of the intrinsic

\footnotetext{
${ }^{2}$ Or, conversely, high energy resolution $\delta \omega$ since $\delta \omega \propto 1 / \tau$.
} 
signal contrast, due to geometrical factors owing to the sample shape (a $3 \mathrm{~mm}$ diameter cylinder in our case), we have used expressions given in Ref. 16. Finally, with straightforward data treatment, we obtained the spectroscopic results detailed in main text.

\section{MEAN-FIELD CALCULATIONS}

Mean-field calculation of the thermal evolution of ordered magnetic moment is performed with the help of expressions given by Johnston 17 . For obtaining the reduced moment $\bar{m}(T)=$ $m(T) / m(T \rightarrow 0)$, we numerically solve the equality

$$
\bar{m}=B_{\mathrm{S}}(y)
$$

using the modified Brillouin function

$$
B_{\mathrm{S}}(y)=\frac{(2 S+1) \cdot \operatorname{coth}[(2 S+1) \cdot y / 2]-\operatorname{coth}[y / 2]}{2 S}
$$

with

$$
y=\frac{3 \bar{m}}{(S+1) \cdot T / T_{\mathrm{N}}} .
$$

In order to compare calculated and measured $\bar{m}(T)$, we multiply $\bar{m}(T)$ from Eq. 10 by a factor $\sqrt{1-f(T)}$ where $f$ is the experimental fluctuating fraction. Such a scaling translates the fact that neutron diffraction is a measure of the elastic (energy integrated) scattering function $\mathcal{S}(Q)=$ $\int \mathcal{S}(Q, \omega) d \omega$ which is itself proportional to the square of the ordered magnetic moment. As shown in Fig. 3a of main text, assuming a local conservation law for the Mn moment and taking an effective spin $S \simeq 0.93$ (corresponding to the saturated value at low temperature $m_{0} \simeq 1.86 \mu_{\mathrm{B}}$ ) yields an excellent agreement between calculation and experiment.

* nicolas.martin@cea.fr

1 A. Tsvyashchenko, V. Sidorov, L. Fomicheva, V. Krasnorussky, R. Sadykov, J. Thompson, K. Gofryk, F. Ronning, and V. Ivanov, in Magnetism and Magnetic Materials V (2012), vol. 190 of Solid State Phenomena, pp. 225-228.

2 M. Deutsch, O. L. Makarova, T. C. Hansen, M. T. Fernandez-Diaz, V. A. Sidorov, A. V. Tsvyashchenko, L. N. Fomicheva, F. Porcher, S. Petit, K. Koepernik, et al., Phys. Rev. B 89, 180407(R) (2014).

3 M. Deutsch, P. Bonville, A. V. Tsvyashchenko, L. N. Fomicheva, F. Porcher, F. Damay, S. Petit, and I. Mirebeau, Phys. Rev. B 90, 144401 (2014). 
4 N. Martin, M. Deutsch, F. Bert, D. Andreica, A. Amato, P. Bonfà, R. De Renzi, U. K. Rößler, P. Bonville, L. N. Fomicheva, et al., Phys. Rev. B 93, 174405 (2016).

${ }^{5}$ N. Martin, M. Deutsch, J.-P. Itié, J.-P. Rueff, U. K. Rössler, K. Koepernik, L. N. Fomicheva, A. V. Tsvyashchenko, and I. Mirebeau, Phys. Rev. B 93, 214404 (2016).

6 N. Kanazawa, Y. Onose, T. Arima, D. Okuyama, K. Ohoyama, S. Wakimoto, K. Kakurai, S. Ishiwata, and Y. Tokura, Phys. Rev. Lett. 106, 156603 (2011).

7 R. Viennois, C. Reibel, D. Ravot, R. Debord, and S. Pailhès, EPL (Europhysics Letters) 111, 17008 (2015).

8 G. Chaboussant, S. Désert, P. Lavie, and A. Brûlet, J. Phys.: Conf. Series 340, 012002 (2012).

9 E. Altynbaev, S.-A. Siegfried, V. Dyadkin, E. Moskvin, D. Menzel, A. Heinemann, C. Dewhurst, L. Fomicheva, A. Tsvyashchenko, and S. Grigoriev, Phys. Rev. B 90, 174420 (2014).

10 B. Hammouda and D. F. R. Mildner, J. of Appl. Cryst. 40, 250 (2007).

11 R. Gähler, R. Golub, and T. Keller, Physica B 180, 899 (1992).

12 J. Kindervater, S. Säubert, and P. Böni, Phys. Rev. B 95, 014429 (2017).

13 Kindervater, J., Martin, N., Häußler, W., Krautloher, M., Fuchs, C., Mühlbauer, S., Lim, J.A., Blackburn, E., Böni, P., and Pfleiderer, C., EPJ Web of Conferences 83, 03008 (2015).

14 W. Häussler and U. Schmidt, Phys. Chem. Chem. Phys. 7, 1245 (2005).

15 C. Franz and T. Schröder, Journal of Large-Scale Research Facilities 1, A14 (2015).

16 N. Martin, Nuclear Inst. and Methods in Physics Research, A 882, 11 (2018).

17 D. C. Johnston, Phys. Rev. B 91, 064427 (2015). 